High Energy Phenomena in Relativistic Outflows III (HEPRO III)

International Journal of Modern Physics: Conference Series

Vol. 8 (2012) 253-258

(C) World Scientific Publishing Company

DOI: $10.1142 / \mathrm{S} 2010194512004679$

\title{
JETS FROM STELLAR TIDAL DISRUPTIONS BY SUPERMASSIVE BLACK HOLES
}

\author{
DIMITRIOS GIANNIOS \\ Department of Astrophysical Sciences, Peyton Hall, Princeton University, \\ Princeton, NJ 08544, USA \\ giannios@astro.princeton.edu \\ BRIAN D. METZGER \\ Department of Astrophysical Sciences, Peyton Hall, Princeton University, \\ Princeton, NJ 08544, USA \\ bmetzger@astro.princeton.edu
}

\begin{abstract}
The tidal disruption of a star by a supermassive black hole provides us with unique information for otherwise dormant galactic nuclei. It has long been predicted that the disruption will be accompanied by a thermal 'flare', powered by the accretion of stellar debris. Recently, we proposed that a modest fraction of the accretion power can be channeled into a relativistic jet. We showed that, even if the jet is not pointing at our direction, the interactions of the jet with the interstellar medium can power a bright radio-IR transient. Recent transients discovered by Swift have all the expected characteristics of a new-born jet powered by the tidal disruption of a star. The evidence is strong that we are witnessing a most direct verification of the our proposal with the transient jet pointing directly at us. Upcoming radio transient surveys can independently discover numerous disruptions, complimenting searches at other wavelengths. Tidal disruptions can probe the physics of jet formation under relatively clean conditions, in which the flow parameters are independently constrained.
\end{abstract}

Keywords: black hole physics - galaxies: nuclei.

\section{Introduction}

Supermassive black holes (SMBHs) are most easily studied when they accrete at high rates for extended periods of time and power active galactic nuclei (AGN). However, the majority of galactic nuclei are relatively quiet. With the exception of Sgr $\mathrm{A}^{\star}$ and a handful of nearby low luminosity AGN, quiescent SMBHs are difficult to study. Even in bright AGN, obtaining a detailed understanding of the accretion process is hindered by our incomplete knowledge of the environment that supplies the gas and sets the accretion rate and the magnetic field strength.

A rare glimpse into the properties of an otherwise quiescent SMBHs is afforded when a star passes sufficiently close that it is torn apart by the SMBH's tidal gravitational field. ${ }^{1,2}$ Analytic estimates and numerical calculations show that the process of disruption leaves a significant fraction of the shredded star gravitationally bound 
to the black hole. ${ }^{2,3}$ The accretion of this stellar debris has long been predicted to power a thermal 'flare' at optical, UV, and X-ray wavelengths that lasts for months to years. ${ }^{4,5}$ Several such detections have been claimed. ${ }^{6,7}$

My talk focuses on the Ref. 8 suggestion that a modest fraction of the accretion power in a tidal disrupton event may be used to launch a relativistic jet and on the apparent observational verification of our claim in the recent transients Swift J164449.3+573451 (hereafter J1644) and Swift J2058.4+0516 (hereafter J2058).

\section{The tidal disruption}

Disruption occurs when the tidal force applied by the SMBH overcomes the self gravity of the star. This occurs when the radius of orbital pericenter $R_{\mathrm{p}}$ is less than $R_{\mathrm{t}} \simeq\left(M_{\mathrm{BH}} / M_{\star}\right)^{1 / 3} R_{\star}$, where $M_{\star}$ and $R_{\star}$ are the stellar mass and radius, respectively. Disruption unbinds $\sim 1 / 2$ of the stellar debris from the system. ${ }^{2}$ The rest of the mass is placed onto highly eccentric orbits, which return it to the vicinity of the black hole on a wide range of timescales ${ }^{9}$. The most tightly bound material returns on a timescale ${ }^{5}$

$$
t_{\text {fallback }} \simeq \frac{2 \pi}{6^{3 / 2}}\left(\frac{R_{\mathrm{p}}}{R_{\star}}\right)^{3 / 2}\left(\frac{R_{\mathrm{p}}^{3}}{G M_{\mathrm{BH}}}\right)^{1 / 2} \simeq 5\left(\frac{M_{\mathrm{BH}}}{10^{7} M_{\odot}}\right)^{5 / 2} \text { days },
$$

where we consider a solar-type $\operatorname{star}\left(R_{\star}=R_{\odot}\right)$ with a pericenter distance $R_{\mathrm{p}}=$ $6 G M_{\mathrm{BH}} / c^{2} \lesssim R_{\mathrm{t}}$.

As material returns to the SMBH, it shocks on itself, circularizes, and accretes ${ }^{10}$. The accretion rate peaks at $t \sim t_{\text {fallback }}$ and declines $\propto t^{-5 / 3}$ thereafter. $^{2}$

\subsection{Relativistic Jet Production}

Black hole accretion is often accompanied by powerful, collimated relativistic outflows. A comparison of the disk luminosity and jet power of blazars, for instance, shows that a substantial fraction of the accretion power goes into relativistic jets (see, e.g., see Ref. 11). A similar correlation between jet and accretion power is observed in elliptical galaxies. ${ }^{12}$

The total energy released by the accretion of a solar mass star is $E_{\text {acc }} \sim 10^{53} \mathrm{erg}$ for an assumed accretion efficiency $\sim 0.1$. The fraction of this energy $\epsilon_{\mathrm{j}}$ placed into relativistic ejecta is highly uncertain and could vary between disruption events. We leave $\epsilon_{\mathrm{j}}$ as a free parameter in our calculations, but adopt a fiducial value $\epsilon_{\mathrm{j}}=0.01$. The total energy of the jet is thus $E_{\mathrm{j}}=\epsilon_{\mathrm{j}} E_{\mathrm{acc}} \sim 10^{51}\left(\epsilon_{\mathrm{j}} / 0.01\right)$ erg, similar to that of a supernova or gamma-ray burst (GRB). We furthermore assume that the jet has an opening angle $\theta_{\mathrm{j}} \approx 0.1$ and a bulk Lorentz factor $\Gamma_{\mathrm{j}}=10$, values typical of AGN jets. The event may last for weeks $t_{\mathrm{j}} \sim t_{\text {fallback }} \sim 10^{6}-10^{7} \mathrm{~s}$.

\section{Jet-ISM interaction}

Jets from AGN are long-lived and propagate to kpc-Mpc distances before dissipating their bulk energy (powering e.g. giant radio lobes). By contrast, any relativistic 

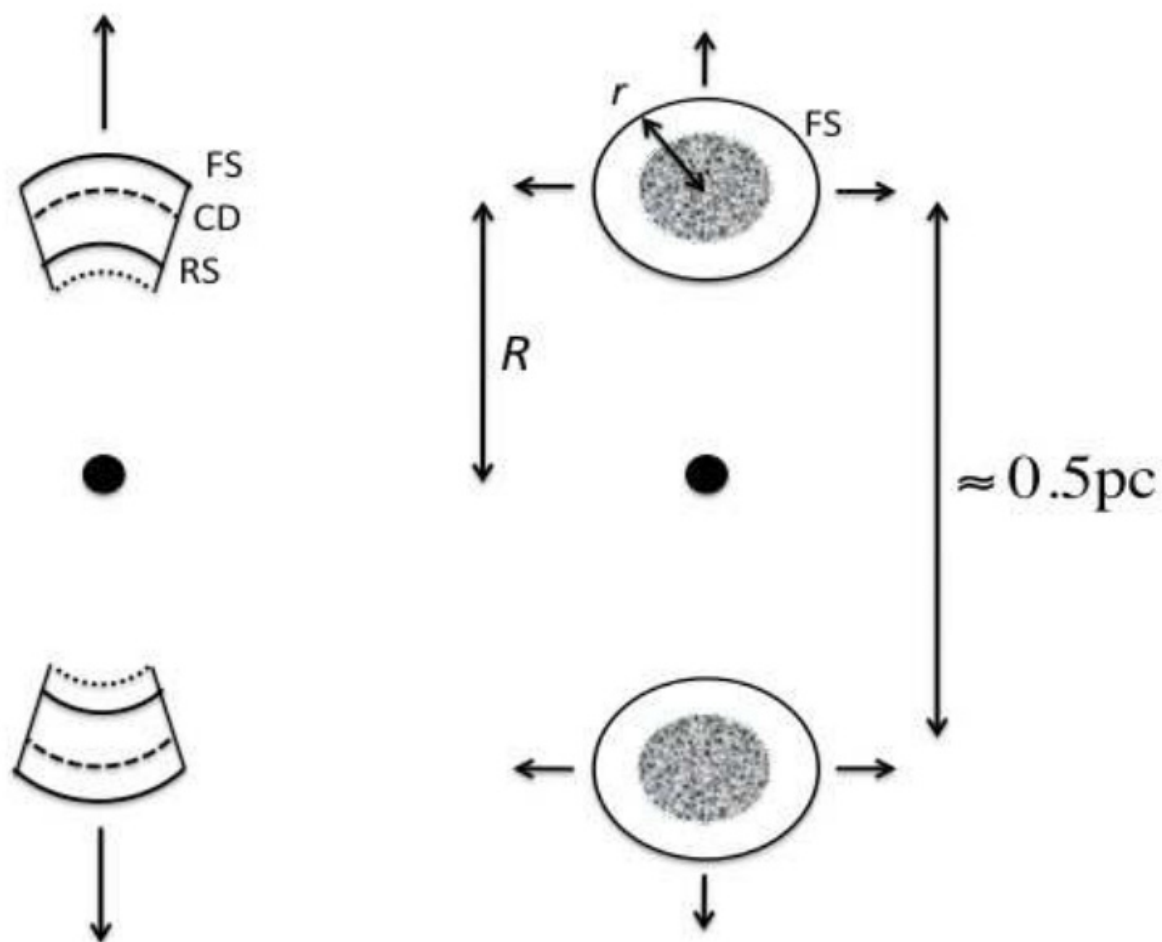

Relativistic Phase

Newtonian Phase
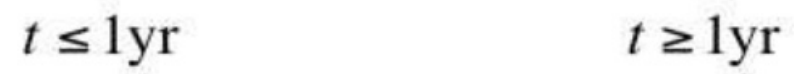

Fig. 1. Sketch of the ultra-relativistic (left) and Newtonian (right) stages of jet deceleration from Ref 8 . Once slowed to non-relativistic speeds, each of the two jet lobes expands quasi-spherically.

outflows from tidal disruption are transient and hence decelerate via interaction with the interstellar medium (ISM) at a much smaller distance $\lesssim$ pc. As the jet drives a forward shock (FS) into the ISM, a reverse shock (RS) propagates through the ejecta, slowing it down. As discussed below, once the reverse shock crosses the jet, the entire configuration (swept-up ISM and shocked ejecta) has been slowed to mildly relativistic speeds. After this point, the flow relaxes into a quasi-spherical, non-relativistic Sedov phase (see Fig. 1).

The interaction between a relativistic jet and the ISM has been studied analytically in Ref. 13. While the reverse shock crosses the ejecta, the shocked fluid decelerates as $\Gamma_{\mathrm{sh}}(R) \simeq \Gamma_{\mathrm{cr}}\left(R / R_{\mathrm{cr}}\right)^{-1 / 2}$, where w $^{\mathrm{a}}$

$$
R_{\mathrm{cr}} \simeq 2 \Gamma_{\mathrm{cr}}^{2} c t_{\mathrm{j}} \approx 7 \times 10^{17}\left(\frac{\epsilon_{\mathrm{j}}}{0.01}\right)^{1 / 4}\left(\frac{n_{\mathrm{ISM}}}{10 \mathrm{~cm}^{-3}}\right)^{-1 / 4}\left(\frac{t_{\mathrm{j}}}{10^{7} \mathrm{~s}}\right)^{1 / 4} \mathrm{~cm}
$$


is the radius at which the reverse shock fully crosses the ejecta, and

$$
\Gamma_{\mathrm{cr}}=\left(\frac{E_{\mathrm{iso}}}{64 \pi n_{\mathrm{ISM}} m_{p} c^{5} t_{\mathrm{j}}^{3}}\right)^{1 / 8} \simeq 1.1\left(\frac{\epsilon_{\mathrm{j}}}{0.01}\right)^{1 / 8}\left(\frac{n_{\mathrm{ISM}}}{10 \mathrm{~cm}^{-3}}\right)^{-1 / 8}\left(\frac{t_{\mathrm{j}}}{10^{7} \mathrm{~s}}\right)^{-3 / 8}
$$

is the Lorentz factor at $R \sim R_{\mathrm{cr}}$. Here $E_{\mathrm{iso}}=E_{\mathrm{j}} f_{\mathrm{b}}^{-1}$ is the 'isotropic' jet energy and $f_{\mathrm{b}} \equiv \theta_{\mathrm{j}}^{2} / 2$ is the jet beaming fraction. We scale the ISM density to $\sim 10 \mathrm{~cm}^{-3}$.

Equations (2) and (3) show that the jet decelerates to a mildly relativistic velocity at $R_{\mathrm{cr}} \sim 0.1-1 \mathrm{pc}$ on a timescale $t_{\mathrm{cr}} \sim R_{\mathrm{cr}} / \beta_{\mathrm{cr}} c \sim \mathrm{a}$ few years. After the RS has crossed the jet, the fluid expands mildly relativistically. After a transient phase the outflow along each jet head relaxes into a quasi-spherical expansion.

In calculating the synchrotron emission from the forward and reverse shocks, we make several standard assumptions regarding the shock microphysics, as motivated by the phenomenology of GRB afterglows (see Ref. 15 for details). First, we assume that a fraction $\epsilon_{e} \sim 0.1, \epsilon_{B} \sim 10^{-2}$ of the energy dissipated at the shock is used to accelerate relativistic electrons and amplify magnetic fields, respectively. We assume the electron distribution is a power-law with index $p \sim 2.5$.

In general the axis of the jet is directed an angle $\theta_{\text {obs }} \sim 1 \mathrm{rad}$ with respect to the line of sight. Emission is weak during the initial relativistic stage because the luminosity is Doppler deboosted by a factor $\propto \delta^{4}$, where $\delta=\Gamma_{\mathrm{sh}}^{-1}\left(1-\beta_{\mathrm{sh}} \cos \theta_{\mathrm{obs}}\right)^{-1} \sim$ $2 / \Gamma_{\text {sh }} \ll 1$. Emission peaks when the RS passes entirely through the ejecta ( $t \sim t_{\text {peak }} \sim t_{\text {cr }}$ ) because a large fraction of the jet energy is dissipated around this time and the expansion has become mildly relativistic $(\delta \sim 1)$.

Figure 2 shows an example of the spectrum of the RS emission at $t \approx t_{\text {peak }} \sim t_{\text {cr }}$ for the reference values of the parameters. In addition to the synchrotron peak frequency at $\nu_{\mathrm{m}} \approx 30 \mathrm{GHz}$, the two other breaks denote the self-absorption and cooling frequencies. In Figure 2 we plot for comparison an estimate of the contemporaneous emission from the accretion disk. The disk emission peaks in the Far-UV, emission from the jet dominates at radio-IR wavelengths. Note that if the jet is modestly energetic $\left(\epsilon_{\mathrm{j}} \sim 10^{-3}-10^{-2}\right)$, the predicted flux $\sim 0.1-1 \mathrm{mJy}$ is well within the sensitivity of current radio telescopes at $\sim$ Gpc distances similar to those of candidate disruption events.

\section{Relativistic tidal disruption candidates}

Swift J1644 was detected by the Swift/BAT on March 25, 2011. Subsequent imaging localized the event to within $\lesssim 100 \mathrm{pc}$ of the center of a galaxy at redshift $z \simeq$ 0.353. ${ }^{16}$ The coincidence of Swift J1644 with the galactic nucleus and the lack of any previous GRBs with similar luminosity or duration, suggest that Swift J1644 most likely originated from a rapid onset of accretion onto a SMBH. ${ }^{17,18,19}$ An X-ray transient with similar properties, Swift J2058, was reported in Ref. 20.

Lack of prior activity at X-ray or radio wavelengths, ${ }^{16}$ suggest that the rapid increase in the accretion rate responsible for Swift J1644 and Swift J2058 cannot result from gas entering the sphere of influence of the SMBH (for instance from 


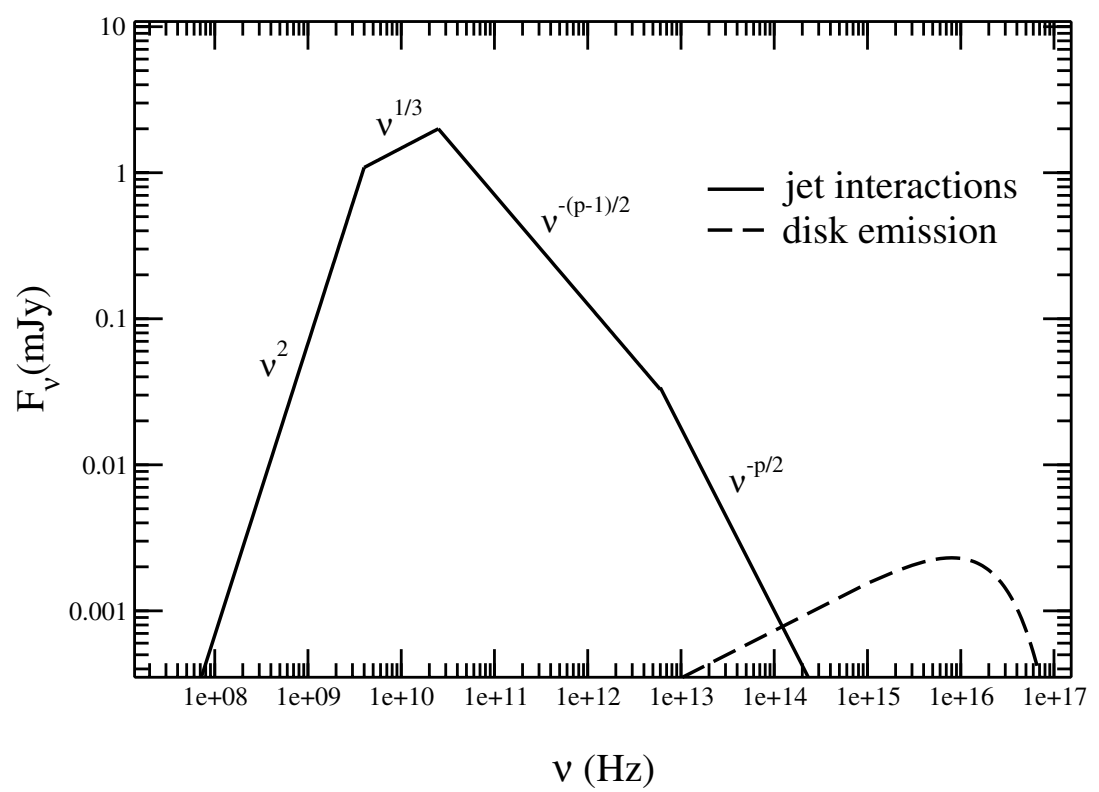

Fig. 2. Predicted synchrotron spectrum (solid line) at the time of peak emission $t_{\text {peak }} \sim t_{\text {cr }} \sim 1$ year from the tidal disruption of a solar mass star by a $10^{7} M_{\odot}$ black hole at a distance $D=1$ Gpc (figure adopted from Ref 8). The dashed line shows an estimate of the thermal emission from the disk at a similar epoch.

the destruction of a passing molecular cloud), since this would require a timescale $\sim r_{\text {soi }} / \sigma>10^{4}$ yr to appreciably alter the accretion rate near the horizon, where $r_{\text {soi }} \sim 1 \mathrm{pc}$ is the radius of the sphere of influence and $\sigma \sim 100 \mathrm{~km} \mathrm{~s}^{-1}$ is a typical bulge velocity dispersion. A more plausible source of such a rapid onset of accretion is the tidal disruption of a star by a SMBH.

The rate of these transients can be estimated by the fact that 2 events have been discovered over the lifetime of Swift out to redshift $z \sim 1 .{ }^{20}$ The observed rate per galaxy is $\sim 10^{-10} \mathrm{yr}^{-1}$. Taking into account that these events are probably beamed into a fraction of $f_{\mathrm{b}} \sim 10^{-3}$ of the sky ${ }^{21}$, the true rate per galaxy may be closer to $\sim 10^{-7} \mathrm{yr}^{-1}$. Compared to the estimated rate of tidal disruption events ${ }^{6}$, it appears that only $\sim$ a few $\%$ of stellar tidal disruptions are launching powerful jets.

\section{Discussion}

In analogy to other accreting black hole systems such as AGN and X-ray binaries, some tidal disruptions of a star by a SMBH appear to be accompanied by a powerful, relativistic jet. We have shown that the interaction of such a jet with the ambient ISM produces a bright radio-infrared transient. These events are, therefore, useful 
probes of the gas density in distant galactic nuclei. ${ }^{21}$ Even when the jet is not pointing at us, radio transients from tidal disruption jets may be detected blindly with upcoming radio transient surveys at a high rate. ${ }^{8}$

Tidal disruptions provide a unique venue to study the physics of accretion, and its connection to jet formation, under conditions in which the 'boundary conditions' of the flow are relatively well-determined. Because the magnetic flux of the disrupted star is insufficient to drive a powerful jet, the detection of tidal disruption jets may favor the hypothesis that locally-generated fields are responsible for jet (although numerical work fails to produce jets under such conditions ${ }^{22}$ ). Furthermore, since only a fraction of (otherwise similar) tidal disruptions shows evidence for a jet, it would indicate that a second parameter in addition to the accretion rate (the black hole spin?) controls the jet strength.

\section{Acknowledgments}

DG acknowledges support from the Lyman Spitzer, Jr. Fellowship awarded by the Department of Astrophysical Sciences at Princeton University. BDM is supported by NASA through Einstein Postdoctoral Fellowship grant number PF9-00065 awarded by the Chandra X-ray Center, which is operated by the Smithsonian Astrophysical Observatory for NASA under contract NAS8-03060.

\section{References}

1. J. G. Hills, Nature 254, 295 (1975).

2. M. J. Rees, Nature 333, 523 (1988).

3. S. Ayal, M. Livio and T. Piran, Astroph. J. 545, 772 (2000).

4. A. Ulmer, Astroph. J. 514, 180 (1999).

5. L. E. Strubbe and E. Quataert, Mon. Not. Roy. Astron. Soc. 400, 2070 (2009).

6. P. Esquej et al., Astron. Astroph. 489, 543 (2008).

7. S. Gezari et al., Astroph. J. 698, 1367 (2009).

8. D. Giannios and B. D. Metzger, Mon. Not. Roy. Astron. Soc. 416, 2102 (2011).

9. C. R. Evans and C. S. Kochanek, Astroph. J. Lett. 346, L13 (1989).

10. C. S. Kochanek, Astroph. J. 422, 508 (1994).

11. G. Ghisellini, L. Maraschi and F. Tavecchio, Mon. Not. Roy. Astron. Soc. 396, L105 (2009).

12. E. Churazov et al., Mon. Not. Roy. Astron. Soc. 332, 729 (2002).

13. R. Sari and T. Piran, Astroph. J. Lett. 455, L143 (1995).

14. D. Giannios, P. Mimica and M. A. Aloy, Astron. Astroph. 478, 747 (2008).

15. R. Sari, T. Piran and R. Narayan, Astroph. J. Lett. 497, L17 (1998).

16. A. J. Levan et al., Science 333, 199 (2011).

17. J. S. Bloom et al., Science 333, 203 (2011).

18. D. N. Burrows et al., Nature 476, 421 (2011).

19. B. A. Zauderer et al., Nature 476, 425 (2011).

20. S. Cenko et al., 2011, (arXiv:1107.5307).

21. B. D. Metzger, D. Giannios and P. Mimica, 2011, [arXiv:1110.1111].

22. S. S. Komissarov and M. V. Barkov, Mon. Not. Roy. Astron. Soc. 397, 1153 (2009). 